\title{
Radio variability properties for sources at multi-frequencies
}

\author{
Y. Liu, J. H. Fan and H. G. Wang \\ Center for Astrophysics, Guangzhou University, Guangzhou 510006, China \\ email: Pinux@pku.org.cn
}

\begin{abstract}
Using three radio databases of the university of Michigan Radio Astronomy Observatory (UMRAO), the National Radio Astronomy Observatory (NRAO), and the Metsähovi Observatory, we analyzed the radio light curves by the power spectral analysis and CLEANest method in search for possible periodicity. Multiple periods are found in some sources at multifrequencies. We adopted the wavelets for period analysis of unevenly sampled time series to search for the evolution of the parameters (period, amplitude) of possible periodicities. Some possible variation of these parameters are found.
\end{abstract}

Keywords. galaxies: blazars - radio continuum - general methods: data analysis

\section{Introduction}

The nature of the central engine of blazars and other classes of active galactic nuclei (AGNs) is still an open problem. Blazars' light curves were generated by using the data of their monitoring programmes which have yielded very valuable information about the mechanisms operating in these sources and important implications for quasar modeling (Fan et al. 1998). In the last about two decades, optical monitoring programmes of blazars and other classes of AGNs have been conducted extensively by many groups around the globe, and blazars were reported to display the flux variability on the diverse time scales ranging from a few minutes to hours, to days, to months and even more than 10 years (Fan 2005). The variability time scale on years gives the long term variation information in the source and the important tool to predict for further outburst time. Fan et al. (2007) adopted power spectral analysis method to search for possible periodicity in the database of UMRAO, the radio sources dispaly astrophysically meaningful periodicity ranging from 2.2 to 20.8 years in their light curves at the three frequencies.

\section{Method}

In the case of unevenly sampled time series analysis, irregular spacing introduces myriad complications into the Fourier transform. It can alter the peak frequency (slightly) and amplitude (greatly), even introduce extremely large false peaks. Many attempts have been made to deal with these problems. A superior technique is the date compensated discrete Fourier transform (DCDFT) and CLEANest method (Foster 1995). The wavelet transform shows great promise as a method for period analysis in time series, particularly for detecting the time evolution of the parameters (period, amplitude, phase) describing periodic and pseudo-periodic signals. In the case of unevenly sampled time series, the weighted wavelet Z-transform (WWZ) can be used to detect, and especially to quantify, periodic and pseudo-periodic signals (Foster 1996). Analysis results for the $8.0 \mathrm{GHz}$ light curve of OJ 287 are shown in Figs. 1, 2 and 3 (Liu et al. 2007, in prep.). 


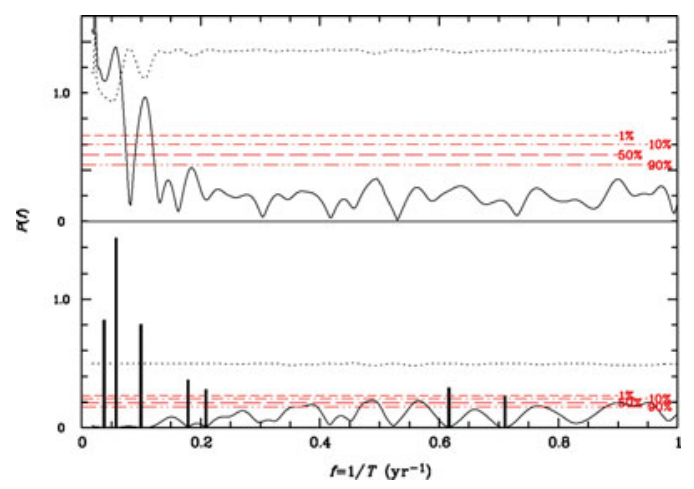

Figure 1. DCDFT and the CLEANest results for the $8.0 \mathrm{GHz}$ light curve of OJ 287. Upper panel: DCDFT result of the adopted light curve; Lower panel: corresponding CLEANest spectrum, where seven frequency components (spikes) and the residual spectrum are shown; Dotted curves: square deviation at different frequencies. Various significance levels are marked.

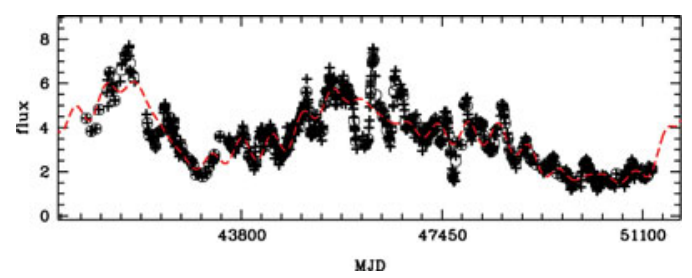

Figure 2. The 8.0 GHz light curve of OJ 287. The dashed curves are the theoretical results obtained by using seven CLEANest components.

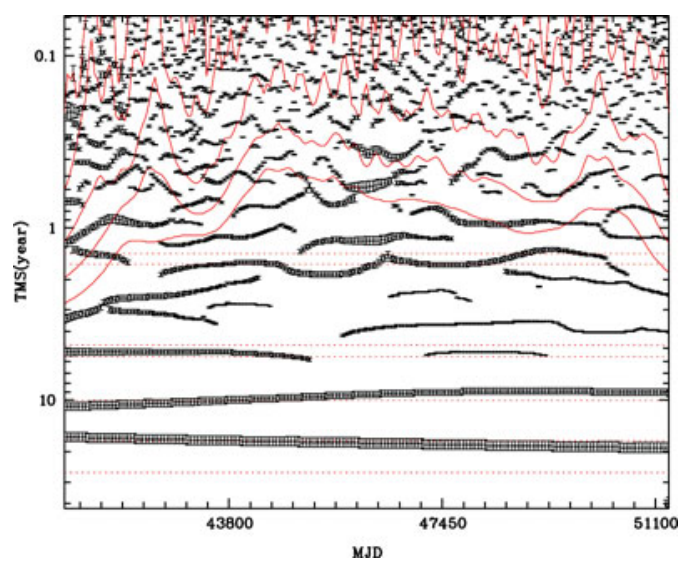

Figure 3. WWZ for the $8.0 \mathrm{GHz}$ light curve of OJ287. The width of the dotted curve represents the amplitude of signal. Seven CLEANest frequency components (dotted lines) are shown. Various effective numbers (Foster 1996) are marked (solid curves).

\section{References}

Fan, J. H., Xie, G. Z., Pecontal, E., et al. 1998, ApJ 507, 173

Fan, J. H. 2005, A\&A 436, 799

Fan, J. H., Liu, Y., Yuan, Y. H., et al. 2007, A\&\&A 462, 547

Foster, G. 1995, AJ 109, 1889

Foster, G. 1996, AJ 112, 1709 